\title{
Workshop on Experimental and Computational Fracture Mechanics 2020
}

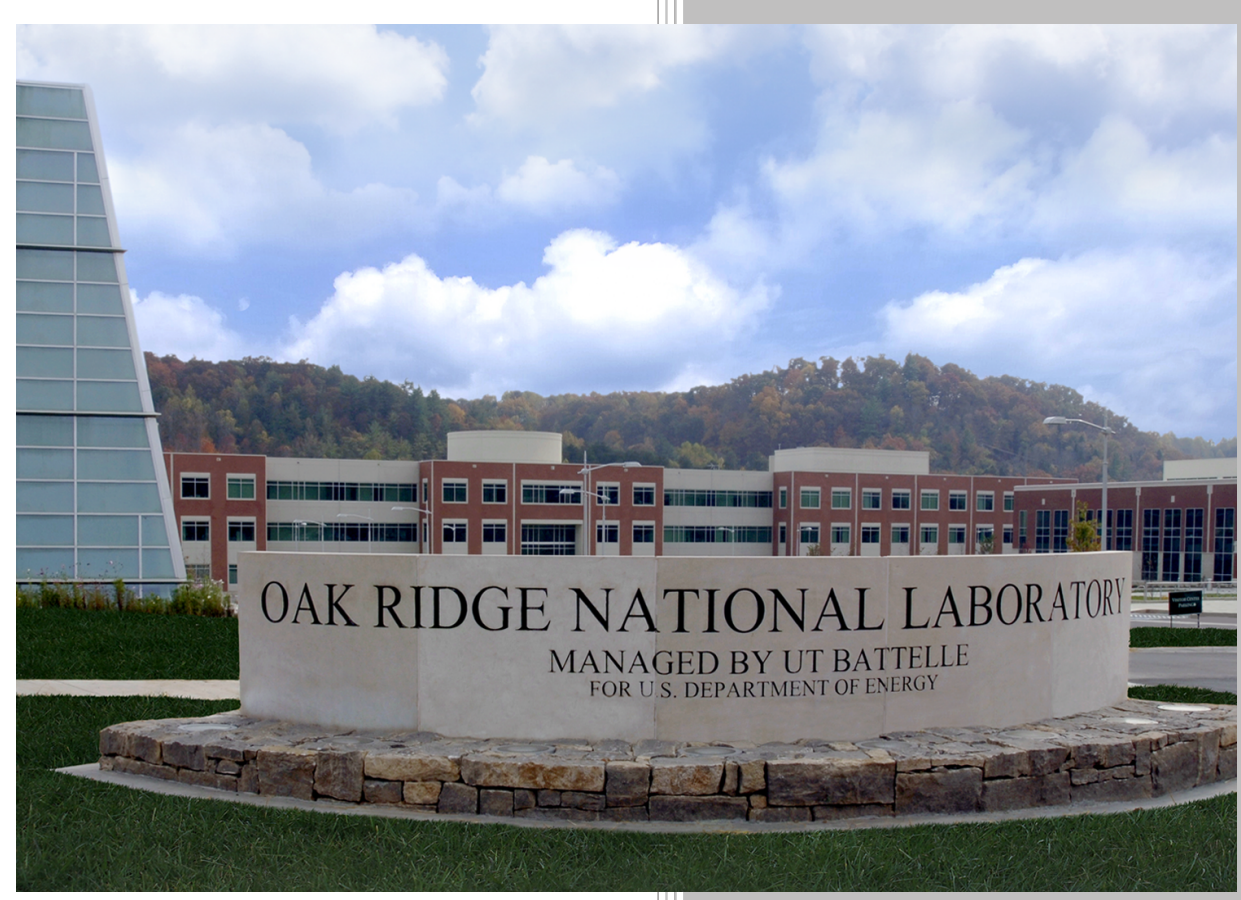

Approved for public release. Distribution is unlimited.

Patrick Diehl

Serge Prudhomme

Pablo Seleson

November 2020 


\section{DOCUMENT AVAILABILITY}

Reports produced after January 1, 1996, are generally available free via US Department of Energy (DOE) SciTech Connect.

Website: www.osti.gov/

Reports produced before January 1, 1996, may be purchased by members of the public from the following source:

National Technical Information Service

5285 Port Royal Road

Springfield, VA 22161

Telephone: 703-605-6000 (1-800-553-6847)

TDD: $703-487-4639$

Fax: 703-605-6900

E-mail: info@ntis.gov

Website: http://classic.ntis.gov/

Reports are available to DOE employees, DOE contractors, Energy Technology Data Exchange representatives, and International Nuclear Information System representatives from the following source:

Office of Scientific and Technical Information

PO Box 62

Oak Ridge, TN 37831

Telephone: 865-576-8401

Fax: 865-576-5728

E-mail: report@osti.gov

Website: http://www.osti.gov/contact.html

This report was prepared as an account of work sponsored by an agency of the United States Government. Neither the United States Government nor any agency thereof, nor any of their employees, makes any warranty, express or implied, or assumes any legal liability or responsibility for the accuracy, completeness, or usefulness of any information, apparatus, product, or process disclosed, or represents that its use would not infringe privately owned rights. Reference herein to any specific commercial product, process, or service by trade name, trademark, manufacturer, or otherwise, does not necessarily constitute or imply its endorsement, recommendation, or favoring by the United States Government or any agency thereof. The views and opinions of authors expressed herein do not necessarily state or reflect those of the United States Government or any agency thereof. 
Computer Science and Mathematics Division

Workshop on Experimental and Computational Fracture Mechanics 2020

\author{
Editors \\ Patrick Diehl, Louisiana State University \\ Serge Prudhomme, Polytechnique Montréal \\ Pablo Seleson, Oak Ridge National Laboratory
}

Date Published: November 2020

\author{
Prepared by \\ OAK RIDGE NATIONAL LABORATORY \\ Oak Ridge, TN 37831-6283 \\ managed by \\ UT-Battelle, LLC \\ for the \\ US DEPARTMENT OF ENERGY \\ under contract DE-AC05-00OR22725
}





\section{CONTENTS}

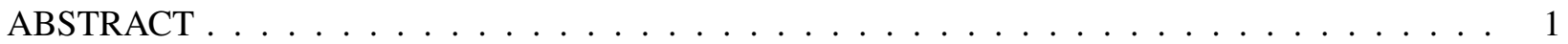

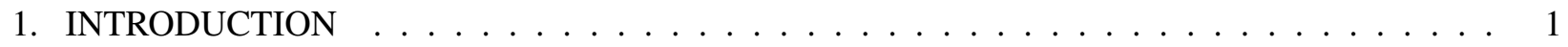

2. BRIEF SUMMARY OF THE WORKSHOP ASPECTS . . . . . . . . . . . . . . . 3

2.1 NONLOCALITY IN PERIDYNAMICS (Stewart Silling) . . . . . . . . . . . . . . . 3

2.2 VARIATIONAL PHASE FIELD MODELING (Thomas Wick) . . . . . . . . . . . . . . 4

2.3 EXPERIMENTS IN FRACTURE MECHANICS (Krishnaswamy Ravi-Chandar) . . . . . 4

2.4 UNCERTAINTY QUANTIFICATION (Johann Guilleminot) $\ldots \ldots \ldots$

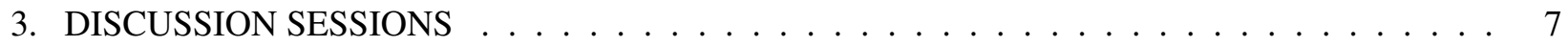

3.1 PERIDYNAMICS (John Dolbow) $\ldots \ldots \ldots \ldots \ldots \ldots \ldots$

3.2 PHASE FIELD MODELING $($ Jay Fineberg $) \ldots \ldots \ldots \ldots \ldots$

3.3 EXPERIMENTAL FRACTURE MECHANICS (Patrick Diehl) . . . . . . . . . . . . . . . 9

3.4 MACHINE LEARNING AND UNCERTAINTY QUANTIFICATION (Serge Prudhomme) . 10

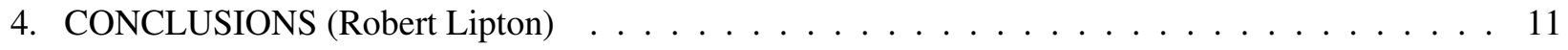

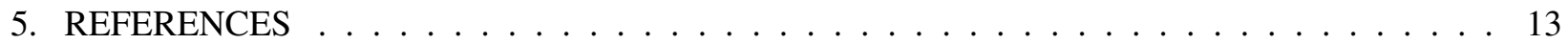

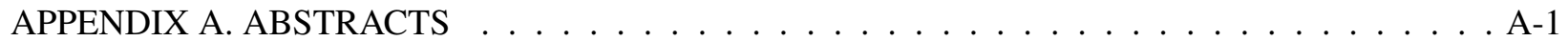

APPENDIX B. PARTICIPANTS' FEEDBACK $\ldots \ldots \ldots \ldots \ldots \ldots \ldots \ldots \ldots$. . . . . . . . . . 



\begin{abstract}
This report describes the objectives, activities, and outcomes of the Workshop on Experimental and Computational Fracture Mechanics, which was held at Louisiana State University in Baton Rouge, Louisiana on February 26-28, 2020.
\end{abstract}

\title{
1. INTRODUCTION
}

The "Workshop on Experimental and Computational Fracture Mechanics: Validating peridynamics and phase field models for fracture prediction and experimental design" was held on February 26-28, 2020 at the LSU Center for Computation \& Technology at Louisiana State University in Baton Rouge, Louisiana.

Comparison of numerical simulations against experimental data is essential for the validation of fracture models in order to gain confidence in their predictability and reliability. Peridynamics and phase field modeling have recently delivered promising results for simulating complex fracture phenomena.

Significant efforts have been carried out in recent years to validate the corresponding fracture models using available experimental data. However, it is still unclear whether the data obtained from current experiments are sufficiently informative to satisfactorily validate models in fracture mechanics. Moreover, it would be interesting to combine numerical tools and experimental design to optimize control parameters in fracture mechanics experiments.

The objectives of the workshop were: (1) to bring together experts in experimental fracture mechanics, peridynamics, and phase field modeling to discuss the state-of-the-art of experimental measurement and computational simulation for fracture mechanics; (2) to promote a dialogue between these communities; and (3) to identify challenges and pathways for robust validation of phase field and peridynamic models as well as integration of experimental and modeling efforts. A brief review of the state-of-the-art for each topic is provided in Section 2.

The workshop was attended by 50 participants in total. It featured 3 keynote lectures, 21 oral presentations, 9 poster presentations, and an industrial talk. The keynote lectures on peridynamics, phase field modeling, and experimental fracture mechanics were presented by Stewart Silling (Sandia National Laboratories), Lallit Anand (Massachusetts Institute of Technology), and Krishnaswamy Ravi-Chandar (The University of Texas at Austin), respectively. The industrial talk was delivered by Danielle Zeng from Ford Motor Company. The abstracts of the oral presentations and poster presentations are available in Appendix A. The workshop also featured four discussion sessions: one on peridynamics led by John Dolbow (Duke University), one on phase field modeling led by Jay Fineberg (The Hebrew University of Jerusalem), one on experimental fracture mechanics led by Patrick Diehl (Louisiana State University), and one on uncertainty quantification and machine learning led by Serge Prudhomme (Polytechnique Montréal). A brief summary of each discussion is provided in Section 3. The participants were asked, ahead of the workshop, to provide feedback on questions suggested by the workshop organizers. The questions, as well as answers given by the workshop participants, are collected in Appendix B.

The workshop was organized by Patrick Diehl (Louisiana State University), Serge Prudhomme (Polytechnique Montréal), and Pablo Seleson (Oak Ridge National Laboratory). The scientific committee was served by Yuri Bazilevs (Brown University), John Dolbow (Duke University), Robert Lipton (Louisiana State University), Krishnaswamy Ravi-Chandar (The University of Texas at Austin), and Stewart Silling (Sandia National Laboratories). The logistics was handled by Jennifer Fontenot and Karen 
Jones (Louisiana State University) and Ruth Hengst and Bethany Roicki (U.S. Association for Computational Mechanics).

The workshop was sponsored by the Technical Thrust Area on Large Scale Structural Systems and Optimal Design of the U.S. Association for Computational Mechanics (USACM), the LSU Center for Computation \& Technology (CCT) at Louisiana State University (LSU), the Oak Ridge National Laboratory (ORNL), the Society for Experimental Mechanics (SEM), and the U.S. National Committee on Theoretical and Applied Mechanics (USNC/TAM). The organizers gratefully acknowledged their support.

\section{Patrick Diehl}

Louisiana State University

Serge Prudhomme

Polytechnique Montréal

\section{Pablo Seleson}

Oak Ridge National Laboratory 


\section{BRIEF SUMMARY OF THE WORKSHOP ASPECTS}

\subsection{NONLOCALITY IN PERIDYNAMICS}

This section discusses the role of nonlocality in continuum mechanics and computational modeling, especially in the peridynamic theory. Nonlocality, for purposes of this discussion, is the presence of an inherent length scale in a mathematical or numerical description, regardless of whether differential, integral, or other types of equations are used.

Nonlocality is not as exotic a concept as people in the mechanics community often assume it to be. For example, the mesh spacing in any discretized form of local partial differential equations (PDEs) necessarily provides a length scale. Since the degrees of freedom interact with each other directly through the discretized equations, the mathematical system is nonlocal.

In many applications of great technological interest, the standard PDEs of continuum mechanics can evolve solutions that become incompatible with the same PDEs. Examples are cracks and dynamic shock waves. Many of the practical methods in common use for mitigating this incompatibility include some aspect of nonlocality. An example is the phase field modeling of fracture, which imposes a length scale over which the phase field variable decays with distance away from a crack surface.

The peridynamic theory goes "all in" on nonlocality: all internal force interactions occur across a finite distance. The resulting equations are integral, rather than differential, allowing cracks to be modeled directly within these fundamental field equations. Although this nonlocality is therefore helpful in modeling fracture, it sometimes gives the impression of introducing unknowable or unmeasurable material properties. For example, researchers in the peridynamics community are frequently asked, "How do you determine the horizon?" (i.e., the cutoff distance for nonlocal interactions).

One way to determine the horizon, as well as a detailed characterization of nonlocal interactions in peridynamics, is to use measured wave dispersion data (Weckner and Silling [2011]). The micromodulus (spring constant) for elastic nonlocal interactions in bonds, as a function of bond length, can be determined directly from dispersion curves.

In either statics or dynamics, the presence of heterogeneities introduces a length scale that determines the micromodulus and length scale in a peridynamic material model. For example, in a composite, the micromodulus as a function of bond length can be fitted to the results of a detailed microscale finite element method (FEM) simulation.

Perhaps surprisingly, nonlocality can be an outcome of homogenization of a local model. Suppose a homogenized displacement is defined to be the weighted average of the displacements in a local model, as determined by a smoothing function. The homogenized displacement turns out to follow the peridynamic nonlocal equation, even though the microscale model was local (Silling [2014]).

In summary, nonlocality is a necessary ingredient in widely used techniques in computational mechanics, such as the FEM, although its appearance is often implicit and goes unrecognized. In peridynamics, an explicit form of nonlocality is derivable from measurable data, such as dispersion curves, as well as in the homogenized description of heterogeneous media.

\section{Stewart Silling}

Sandia National Laboratories 


\subsection{VARIATIONAL PHASE FIELD MODELING}

Variational phase field fracture is a very active research area with research questions in calculus of variations, numerical analysis, and engineering. Current investigations address questions in the further verification of formulations; crack nucleation; constitutive models (energy splittings, ductile fracture, nearly incompressiblity) that are sound in the thermodynamical context; rigorous numerical analysis; adaptive-robust, efficient numerical solution of large-scale problems; and multiphysics phase field fracture applications. In recent review articles (Bourdin and Francfort [2019]; Wu et al. [2020]; Wheeler et al. [2020]), current advancements are summarized. In Bourdin and Francfort [2019], the pioneering authors review more theoretical questions. The authors of $\mathrm{Wu}$ et al. [2020] provide an extensive overall summary. A high-performance parallel-adaptive framework including physics-based-discretizations and various coupling schemes in multiphysics phase field fracture is the subject in Wheeler et al. [2020].

The phase field section of this workshop covered some of the previously mentioned topics. Related talks started with the keynote lecture of Lallit Anand on gradient-damage theory. The challenge of crack nucleation using phase field models has often been subject of converse discussions. Basically, phase field fracture modeling allows for crack nucleation, but quantitative and rigorous understanding is still missing and some recent efforts have been shown by Oscar Lopez-Pamies. Haim Waisman concentrated on thermo-mechanical phase field fracture, while Kerstin Weinberg presented another stress (energy) splitting constitutive equation. Chad Landis and Thomas Wick presented findings in ductile and fatigue fracture as well as nearly incompressible solids and computational convergence analyses, respectively; these talks also pointed to local mesh refinement. In fact, the combination of adaptive methods together with parallel computations are promising (and necessary) techniques in tackling more practical applications, which was exactly the purpose of this workshop, i.e., to bring together experiments, simulations, and theory.

\section{Thomas Wick}

Leibniz University Hannover

\subsection{EXPERIMENTS IN FRACTURE MECHANICS}

Methods for computational simulation of physical phenomena related to the mechanical behavior of materials in general, and fracture behavior in particular, have greatly expanded over the past few decades. Furthermore, the computational power and efficiency in handling very large simulations, encompassing multiscale models, has also increased enormously. As a result of these developments, solutions to almost any model of fracture can be generated rapidly. In such an environment, many researchers expected that expensive experiments would simply become irrelevant or at least of secondary importance-relegated to providing data for comparison to code output; fortunately, this has not come to pass. In fact, experiments have maintained a dual role of significance. First, experiments provide the physical underpinnings to the development of any model that is eventually numerically simulated; in other words, they define the physical problem that has to be formulated. Second, they provide critical observations and measurements of relevant quantities of interest (QoIs) that are to be replicated in the computational simulations.

Advances in automation, control, and high-speed, high-fidelity data acquisition methods, on the one hand, and the development of measurement methodologies using techniques such as digital image correlation, on the other hand, have permitted the exploration of a range of QoIs in comparison to a very limited set of experimental measurements even a decade ago. For example, early measurements in fracture experiments 
were limited either to far-field load vs. load-point displacement variation, where even the location of the crack tip had to be inferred from compliance changes, or to indirect methods such as photoelasticity. Recent advances allow local measurements of field variables such as the displacement or its gradient at high spatial and temporal resolution. These experimental measurements, combined with post-mortem fractographic observations and measurements, provide a wealth of experimental data aimed both at model development and validation.

The energetic basis for quasi-static fracture in linearly elastic materials, founded on Griffith's original ideas from a century ago, has been clearly validated through decades of experimental investigations. The foundations of dynamic fracture are also rooted firmly in the energy balance approach to linearly elastic fracture; theoretical modeling of certain aspects of dynamic fracture related to an observed limiting speed well below the Rayleigh wave speed and the emergence of crack branching continues to pose challenges. While significantly different in their modeling of fracture, both peridynamics and phase field modeling - the themes of this workshop-are able to simulate the growth of both quasi-static and dynamic cracks under arbitrary loading conditions. However, rigorous procedures for calibration of the critical internal parameters of the models - the horizon size, $\delta$, and the regularization length, $l_{0}$, in the peridynamic and phase field models, respectively — have yet to be established. In the context of calibrating and validating the peridynamic and phase field models of fracture in linearly elastic materials, we identify the following challenging examples from the rather vast experimental literature.

Selection of QoIs is an important issue in the process of model validation through quantitative comparison to experiments, particularly in problems related to the evolution of such QoIs. For fracture problems, this should include a combination of global and local quantities; for example, choosing the load-point displacement as the loading control parameter, the response parameters, at a minimum, would be the applied load (global parameter) and the crack tip position (local parameter). Experimental results of global measurements are available for mode I and mixed-mode loading in the literature; for example, there is a large data set for concrete (Gálvez et al. [1998]; Hoover et al. [2013]). Evolution data for crack position is also available in some publications (see, e.g., Pham et al. [2017]; Carpiuc et al. [2018]). A more challenging problem for model validation in two-dimensional cases is associated with the instabilities in the growth of cracks under thermal quenching (Yuse and Sano [1993]; Ronsin et al. [1995]; Yang and Ravi-Chandar [2001]); while both peridynamic and phase field models show the instabilities, the challenge lies in using independently calibrated parameters to predict the instability diagram. Another example of the challenges arising from experimental observations and measurements under quasi-static loading is the case of mixed-mode I plus III; here, the growing crack generates spectacular three-dimensional patterns (see, e.g., Leblond et al. [2011]). There are some attempts at phase field modeling of this problem (Chen et al. [2015]; Pham and Ravi-Chandar [2017]), but not from peridynamics; even qualitatively mimicking observed features in this case appears to be challenging.

The problem of dynamic fracture presents a wealth of experimental data for validation of peridynamic and phase field models of fracture. Experiments investigating various aspects of dynamic crack propagation, both microscopic and macroscopic, have been reported in the literature (see, e.g., Sundaram and Tippur [2018]; Fineberg and Bouchbinder [2015]; Ravi-Chandar and Knauss [1984a,b,c]). These publications have discovered and quantified a rather wide range of interesting phenomena that emerge during dynamic crack propagation - fracture energy increase or dissipation and the associated evolution of crack surface roughness, onset of microbranching instabilities, macroscopic crack branching, oscillatory instability if branching is suppressed, etc. While these represent a large range of QoIs for comparison with simulations, the focus for comparisons with simulations (see, e.g., Borden et al. [2012]; Bobaru and Zhang [2015]) 
appears to have been somewhat limited, restricting attention to qualitative aspects, such as the occurrence of branching and kinked crack growth in the Kalthoff experiment (Kalthoff [2000]). However, the mode I crack responses reported in the literature have numerous QoIs that would provide the ultimate model validation and this needs to be addressed in future work.

This workshop provided an opportunity for exchange of ideas between researchers approaching fracture mechanics experimentally as well as numerically. As the capability of numerical simulation tools improve with time, so does the resolution of modern experimentation; this provides an opportunity to close the loop on validation of computational models and transition them into practical tools for assessment of safety and durability of structures. It is important to follow up on this workshop and to develop these interactions between experimental and computational groups perhaps through joint work where the experimental and computational parts are planned jointly, somewhat along the lines of the Sandia Fracture Challenge for ductile materials.

Krishnaswamy Ravi-Chandar

The University of Texas at Austin

\subsection{UNCERTAINTY QUANTIFICATION}

Uncertainty quantification (UQ) aims at endowing numerical predictions with appropriate measures of confidence, using concepts and tools from probability theory and mathematical statistics. Once an emerging field, it has become a predominant component of predictive science and engineering. UQ essentially covers four intertwined topics, namely:

1. Probabilistic Modeling: this topic is concerned with the representation of model and parametric uncertainties (e.g., through polynomial chaos expansions);

2. Uncertainty Propagation: this component is focused on the propagation of uncertainties through computational models, using intrusive or non-intrusive approaches;

3. Parameter Identification: this topic deals with the calibration of hyperparameters based on limited observables, usually by solving statistical inverse problems (e.g., within a Bayesian framework);

4. Model Validation: this activity aims at assessing the relevance of the models and strategies developed within the topics 1, 2, and 3, considering, e.g., statistical metrics on selected QoIs.

The field has been very active over the past two decades and state-of-the-art technical overviews can be found in Ghanem et al. [2017]. Reviews and applications focusing on materials modeling can be found in Wang and McDowell [2020].

Johann Guilleminot

Duke University 


\section{DISCUSSION SESSIONS}

\subsection{PERIDYNAMICS}

The discussion session covered two main questions:

1. What are the biggest challenges and opportunities in peridynamics fracture modeling?

2. What do we need to experimentally validate peridynamic fracture models?

For the first question, the responses submitted were broken down into science-based challenges and methodological challenges. The list of science-based answers included: crack nucleation, ductile-based fracture, connecting fracture criteria to mechanisms, predicting oscillatory instabilities at high velocities, understanding the form of the cohesive zone and what it depends on, faceting in 3D, and the interaction of crack fronts with rigid or soft particles. The list of methodological challenges included: calibration of material properties, the influence of the nonlocal horizon on the fracture process and physical length scales, boundary conditions in general, enforcing contact conditions on crack faces, extracting crack lengths and speeds in robust ways, demonstrating spatial convergence, and coupling to classical continuum mechanics formulations.

For the second question, three main responses were provided: (i) a collection of carefully documented challenge problems spanning mixed-mode conditions, quasi-brittle behavior, dynamics, etc.; (ii) detailed measurements of fields ahead of a rapidly moving crack tip; and (iii) more tests on materials where Poisson's ratio is 0.25 . This was followed by a discussion of the bond-based version of peridynamics (appropriate for a Poisson's ratio of 0.25 ) vs. the state-based version which is more general. The question was asked as to why the bond-based version appears to be used even for materials for which Poisson's ratio is not 0.25 . The consensus seemed to be that the bond-based version is much less expensive computationally.

There was a brief discussion of what is meant by a cohesive zone and the concept of there being two crack tips. The leading tip can be thought of as the "mathematical" crack tip, normally where the singularity is located. The trailing tip is a "physical" tip at which point no traction is being transmitted between the crack faces. In between is the fracture process zone where tractions are related (in most cohesize zone models) to the separation of the crack surfaces. There was a discussion of how such laws can be incorporated into peridynamic models and the work of Phillippe Geubelle (University of Illinois at Urbana-Champaign) was discussed.

The discussion then focused on the horizon length and how to determine it. There was some notion that it should correspond to the size of the fracture process zone which in some materials can be exceptionally small compared to structural length scales. Finally, there was a discussion of "multiphysics" problems and why there seemed to be the use of conventional continuum models for some aspects of the physics instead of peridynamics for everything. The consensus again seemed to be that while this could be done in general the nonlocality brings additional computational expense.

John Dolbow

Duke University 


\subsection{PHASE FIELD MODELING}

The discussion session covered two main questions:

1. What are the biggest challenges and opportunities in phase field fracture modeling?

2. What do we need to experimentally validate phase field fracture models?

For the first question, the following challenges were raised:

- Incorporation of well-defined nucleation criteria.

- The need to relate the phase field length scale to physical processes. What is experimentally testable?

- Preventing crack reversion: A maximum historical driving energy is energetically inconsistent and may pollute simulation results. Proposed solutions exist; these include inequality-constrained solvers and Lagrange multipliers. Unfortunately, these solutions significantly increase the implementation effort, which might put the phase field method out of reach.

- Computational expense remains an issue, due to the need of sufficient resolution of an often-miniscule regularization length scale.

- There doesn't seem to be a consensus on how best to incorporate phase field models (which typically involve an elliptic PDE) into fully-explicit hydrocodes used for extreme event simulation.

If we, as a community, can overcome the different challenges, the gains may be significant. Additionally, the following opportunities were discussed:

- Predictions of ductile fracture, comparison with damage models and experiments.

- Understanding and predicting crack dynamics within inhomogeneous materials.

- Possibly finding ways to predict the effective fracture energy (or fracture toughness) in heterogeneous materials - and to explore if such a 'simple' scenario even exists. In other words, can the effects of material inhomogeneities be incorporated solely into a simple, phenomenological scalar quantity, such as fracture energy — or do more involved dynamics take place in which the microscale significantly affects the macroscale?

- Phase field models may be useful for comparing crack paths when crack fronts interact with discrete particles/inclusions, which locally break the symmetry of materials.

- Phase field models may give us better understanding of 3D fracture. Can the experimentally observed faceting and nontrivial topology be modeled and understood? How does a crack front behave under large (finite) perturbations? Can the crack front break up into separate, stable, fracture planes?

- This class of models are extremely useful in understanding the fracture of materials that undergo large deformations. Here, there are examples where the classical LEFM framework may break down. How do fracture dynamics/properties change in extremely deformable (e.g., elastomers) materials?

For the second question, there were a number of interesting points raised. As in the discussion of peridynamics, participants were interested in validation of the phase field model predictions in much the same way; either by comparing their results to predictions of various "experimental challenges" or to existing data sets. There is a need for a collection of carefully documented challenge problems spanning a range of ingredients, such as mixed-mode, quasi-brittle (cohesive zone), dynamics, and ductile fracture. 
One problem raised in both the talks and discussion was the question/problem of nucleation in fracture. Any such experimental validation or test has to be relatively "immune" to defect-driven nucleation. In some loading configurations, the onset of fracture and the location of fracture initiation are extremely sensitive to the existence of defects or nearly invisible perturbations. In such cases, any comparison to predictions would be very difficult. Good test cases for dynamic fracture codes would in comparison to well-defined cracks that are already propagating. Comments were made - that encouraged experimentalists to make detailed dynamic data sets (or even movies) publicly available. Theorists requested access to quality high-speed footage of dynamic fracture under controlled experimental conditions.

An additional subject that came up was the structure of the cohesive zone (process zone) that regularizes the singularity that is described successfully by LEFM. Currently, there are a number of heuristic models for the cohesive zone, but very few experiments exist. While the structure of the cohesive zone may well be material-dependent, it is certainly conceivable that different general forms, or "universality classes", of cohesive zones could exist. Understanding these structures may well be a key to both a better "first principles" theory of fracture and less ad-hoc modeling of this important region where fracture actually occurs. A better understanding of the structure (and possible dynamical behavior) of the cohesive zone could be utilized to better select the proper class of models to use to describe fracture (or even different "universal" types of fracture).

Jay Fineberg

The Hebrew University of Jerusalem

\subsection{EXPERIMENTAL FRACTURE MECHANICS}

For this discussion session, the following questions were asked to drive the discussion:

1. What are the biggest challenges and opportunities in experimental fracture research?

2. How fracture modeling could help experimental fracture research?

For the first question, two main challenges were identified. First, most of the available experimental data is two-dimensional and not many three-dimensional data is available. A reason provided for that was that these experiments are just difficult and thus we do not have yet industrial standards, e.g., ASTM or DIN, for such experiments. The peridynamics and phase field communities raised the question about the resolution of three-dimensional experimental measurements. Here, we have a rich spatial but poor temporal resolution, since accruing and reconstructing different states can take from five minutes up to two hours and result in millions or billions of data per snapshot. Second, some materials do not allow direct digital image correlation measurements and a speckle pattern is needed. Here, the design of such a pattern is the challenge. Once a speckle pattern is designed and the QoI can be obtained using digital image correlation, the next challenge arises. Since we cannot obtain digital image correlation measurements everywhere on the specimen, we need to know in advance where the crack initiates and focus the camera on that area. Especially for homogeneous materials, it is very difficult to predict that area and typically a notch is introduced to control where cracks initiate.

For the second question, the following points were emphasized:

- Simulations could be used for sensitivity analysis and quantification of uncertainties.

- The repeatability of experiments was questioned and currently this can throw experiments off. Also, 
there can be material defects or testing conditions which influence the experiment. A common way to account for these is to take a piece of the specimen or the object used in the experiment and perform different tests to characterize the experiment. For example, the Sandia Fracture Challenge (Boyce et al. [2014, 2016]; Kramer et al. [2019]) combines material characterization and fracture tests of the same object.

- What is a reasonable error for experimental fracture mechanics? How to distinguish between error and uncertainty? This depends on the QoI.

- For crack nucleation, the question of how to model material defects was raised, e.g., what kind of distribution is suitable or what kind of pattern should be used? In experiments, we introduce defects, for example, by a razor blade.

- What do experimentalists wish or need from modelers?

- Computation of quantities that are really hard to measure: Homogeneous loading works assuming linear elasticity; however, in most materials there is heterogeneity (due to material defects) that manifests in experiments as random behavior and the QoI pops up randomly. Here, the model and test must go hand in hand and should model material defects.

- A key concept here is "multiphysics" simulations, because quantities are hard to predict and measure in this case.

Patrick Diehl

Louisiana State University

\subsection{MACHINE LEARNING AND UNCERTAINTY QUANTIFICATION}

The discussion session was steered around the following question:

"What are the biggest challenges and opportunities in machine learning and in uncertainty quantification for computational and experimental fracture mechanics?"

Some participants raised concerns about whether machine learning (ML) can be useful to fracture mechanics. Producing large amounts of experimental data and measurements for the training of the ML models can be challenging. Even if one has access to large datasets, one could still argue that the resulting ML models may not have the predictive power that physics-based models have. It was mentioned that the models could actually be predictive within the regions covered by the data but would not be as reliable when extrapolating to scenarios outside of those regions. One question is whether the tools of ML can help us learn about the phenomenological models arising in fracture mechanics. The use of ML, for instance the classification methods, can extract patterns from unstructured data and thus provide partial information about features that are not understood. In that sense, ML could be useful for a better understanding of the underlying physics and could bring insights for experimental design activities. An opportunity in ML could be to employ phase field and peridynamics simulations to generate large datasets for the training of neural network models in order to reduce current simulation times needed by physics-based models.

A general remark was that uncertainty quantification (UQ) is still rarely considered in fracture mechanics. Simulations are mostly deterministic. Possible reasons are that "tracking and integrating all uncertainties across multiple aspects of a fracture experiment starting from material and geometric variability to measured load or displacement variability to methodologies to data extraction is prohibitively expensive" 
and time-consuming, that experimental data may be too scarce to extract reasonable statistics, that "models should be predictive", etc. However, it was recognized that the Bayesian framework can allow one to identify parameters with their probability densities with only a few data samples (e.g., digital image correlation works with five samples). Other opportunities would be to develop fast stochastic solvers and reduced-order models so as to keep simulation times reasonable.

There was a brief discussion of model validation methodologies for fracture mechanics. It is clear that we should consider using tools developed in other fields, such as cross-validation, for better assessment of the calibrated parameters. Challenges include defining a general framework for validation and coming up with reasonable bounds on QoIs in order to decide whether the fracture models are "not invalidated". The Sandia Fracture Challenge was also mentioned in the discussion.

\section{Serge Prudhomme}

Polytechnique Montréal

\section{CONCLUSIONS}

Computational models allowing for the natural emergence of cracks are desirable for capturing the effects of crack physics and for enabling engineering decisions and design. Both peridynamics and phase field modeling provide computational tools developed with this goal in mind. Quantitative and qualitative physical understanding of fracture and damage requires insightful experimental techniques aimed at teasing out the underlying physics. Taken together the computational models coupled with the physical knowledge and validation gleaned from experiments can enable predictive simulation tools for design and unlock a synergy for a deeper understanding of the fracture process.

Over the course of this workshop, presentations from the peridynamics, phase field, and experimental fracture mechanics communities were delivered. The presentations were followed by a daily discussion session. The workshop included a conference dinner with a presentation discussing computational damage mechanics and the associated challenges facing the automotive industry.

A distinctive and synergistic outcome of the workshop was that further integration of experimentation and computation for both quasi-static and dynamic fracture experiments are to be made. Along these lines is the availability of new data from high-fidelity, high-speed videos of the fracture process for use by the computational fracture mechanics community to calibrate their models. This provides opportunities for numerical validation and prediction. Such experimental data as generated will provide a valuable set of benchmarks for the calibration and further development of computational methodologies. Most importantly, the meeting facilitated a distinct and positive step forward for an enhanced coordination between the methodologies of peridynamics, phase field, and experimental fracture mechanics.

\section{Robert Lipton}

Louisiana State University 



\section{REFERENCES}

F. Bobaru and G. Zhang. Why do cracks branch? A peridynamic investigation of dynamic brittle fracture. International Journal of Fracture, 196:59-98, 2015.

M. J. Borden, C. V. Verhoosel, M. A. Scott, T. J. R. Hughes, and C. M. Landis. A phase-field description of dynamic brittle fracture. Computer Methods in Applied Mechanics and Engineering, 217-220:77-95, 2012 .

B. Bourdin and G. A. Francfort. Past and present of variational fracture. SIAM News, 52(9), 2019.

B. L. Boyce, S. L. B. Kramer, H. E. Fang, et al. The Sandia Fracture Challenge: blind round robin predictions of ductile tearing. International Journal of Fracture, 186:5-68, 2014.

B. L. Boyce, S. L. B. Kramer, T. R. Bosiljevac, et al. The second Sandia Fracture Challenge: predictions of ductile failure under quasi-static and moderate-rate dynamic loading. International Journal of Fracture, 198:5-100, 2016.

A. Carpiuc, M. Poncelet, J. Réthoré, and S. Roux. CARPIUC benchmark overview: crack advance, reorientation, propagation and initiation under complex loadings. Advanced Modeling and Simulation in Engineering Sciences, 5:1-15, 2018.

C.-H. Chen, T. Cambonie, V. Lazarus, M. Nicoli, A. J. Pons, and A. Karma. Crack front segmentation and facet coarsening in mixed-mode fracture. Physical Review Letters, 115:265503, 2015.

J. Fineberg and E. Bouchbinder. Recent developments in dynamic fracture: some perspectives. International Journal of Fracture, 196:33-57, 2015.

J. C. Gálvez, M. Elices, G. V. Guinea, and J. Planas. Mixed mode fracture of concrete under proportional and nonproportional loading. International Journal of Fracture, 94:267-284, 1998.

R. Ghanem, D. Higdon, and H. Owhadi, editors. Handbook of Uncertainty Quantification. Springer International Publishing, 2017.

C. G. Hoover, Z. P. Bažant, J. Vorel, R. Wendner, and M. H. Hubler. Comprehensive concrete fracture tests: Description and results. Engineering Fracture Mechanics, 114:92-103, 2013.

J. F. Kalthoff. Modes of dynamic shear failure in solids. International Journal of Fracture, 101:1-31, 2000.

S. L. B. Kramer, A. Jones, A. Mostafa, et al. The third Sandia fracture challenge: predictions of ductile fracture in additively manufactured metal. International Journal of Fracture, 218:5-61, 2019.

J.-B. Leblond, A. Karma, and V. Lazarus. Theoretical analysis of crack front instability in mode I+III. Journal of the Mechanics and Physics of Solids, 59:1872-1887, 2011.

K. H. Pham and K. Ravi-Chandar. The formation and growth of echelon cracks in brittle materials. International Journal of Fracture, 206:229-244, 2017.

K. H. Pham, K. Ravi-Chandar, and C. M. Landis. Experimental validation of a phase-field model for fracture. International Journal of Fracture, 205:83-101, 2017. 
K. Ravi-Chandar and W. G. Knauss. An experimental investigation into dynamic fracture: II. Microstructural aspects. International Journal of Fracture, 26:65-80, 1984a.

K. Ravi-Chandar and W. G. Knauss. An experimental investigation into dynamic fracture: IV. On the interaction of stress waves with propagating cracks. International Journal of Fracture, 26:189-200, 1984b.

K. Ravi-Chandar and W. G. Knauss. An experimental investigation into dynamic fracture: III. On steady-state crack propagation and crack branching. International Journal of Fracture, 26:141-154, $1984 c$.

O. Ronsin, F. Heslot, and B. Perrin. Experimental study of quasistatic brittle crack propagation. Physical Review Letters, 75:2352, 1995.

S. A. Silling. Origin and effect of nonlocality in a composite. Journal of Mechanics of Materials and Structures, 9:245-258, 2014.

B. M. Sundaram and H. V. Tippur. Dynamic fracture of soda-lime glass: A full-field optical investigation of crack initiation, propagation and branching. Journal of the Mechanics and Physics of Solids, 120: 132-153, 2018.

Y. Wang and D. L. McDowell, editors. Uncertainty Quantification in Multiscale Materials Modeling. Woodhead Publishing, 2020.

O. Weckner and S. A. Silling. Determination of nonlocal constitutive equations from phonon dispersion relations. International Journal of Multiscale Computational Engineering, 9:623-634, 2011.

M. F. Wheeler, T. Wick, and S. Lee. IPACS: Integrated Phase-Field Advanced Crack Propagation Simulator. An adaptive, parallel, physics-based-discretization phase-field framework for fracture propagation in porous media. Computer Methods in Applied Mechanics and Engineering, 367:113124, 2020.

J.-Y. Wu, V. P. Nguyen, C. T. Nguyen, D. Sutula, S. Sinaie, and S. P. A. Bordas. Chapter One - Phase-field modeling of fracture. volume 53 of Advances in Applied Mechanics, pages 1-183. Elsevier, 2020.

B. Yang and K. Ravi-Chandar. Crack path instabilities in a quenched glass plate. Journal of the Mechanics and Physics of Solids, 49:91-130, 2001.

A. Yuse and M. Sano. Transition between crack patterns in quenched glass plates. Nature, 362:329-331, 1993. 
APPENDIX A. ABSTRACTS 



\title{
ORAL PRESENTATIONS
}

\section{Integrated Computational Materials Engineering (ICME) development of carbon fiber composites for lightweight vehicles}

\author{
Danielle Zeng \\ Ford Motor Company \\ Co-Authors: P. Seleson, B. Ren, and C. T. Wu
}

Automotive manufacturers use lightweight materials to meet the increasing demands of fuel efficiency. The Carbon Fiber Reinforced Polymer (CFRP) composites, with a density of $1.55 \mathrm{~g} / \mathrm{cm}^{3}$ and a tensile strength of $2000 \mathrm{MPa}$ in the fiber direction, are among the most promising candidates to replace the metals currently used for structural components. It is important to note that the performance of carbon fiber composites is determined not only by the component design, but also the manufacturing processes. In this talk, the focus is on the application of an Integrated Computational Materials Engineering (ICME) approach to the structural composite design. A suite of predictive models is developed to link materials design, manufacturing process and final performance to enable optimal design and manufacturing of CFRP components for automotive vehicles.

One of the greatest challenges for successfully applying the ICME approach to CF composites is how to accurately simulate the different failure modes during crash scenarios. Especially, the traditional thin shell model in finite element simulation has difficulty in capturing the delamination behavior during complex loading conditions. Recently, a discontinuous Galerkin weak form for bond-based peridynamic models is developed for composite modeling through the collaboration among ORNL, LSTC and Ford. The accuracy and computational efficiency of the developed model for delamination modeling is demonstrated through simulating a dynamic bending test of a laminate structure. 


\title{
Nonlocality in peridynamics
}

\author{
Stewart Silling \\ Sandia National Laboratories
}

Nonlocality is an essential feature of the peridynamic model of solid mechanics, which treats all internal forces as acting through finite distances. This nonlocality, which avoids the need to evaluate partial derivatives of the deformation, helps peridynamics treat singularities such as evolving cracks within its basic field equations.

In this talk I will offer a perspective on the significance and effect of nonlocality in the peridynamic continuum model and other theories. Nonlocality offers a mathematical tool to treat certain physical effects such as wave dispersion and attenuation in more generality than is possible in the local theory. It enables the modeling of interesting phenomena such as solitary waves, as well as fracture and fragmentation. It provides a natural compatibility of peridynamics with nanoscale long-range forces. On the other hand, nonlocality is sometimes inconvenient in macroscale simulations, for example by creating surface effects in material properties.

Is nonlocality real? Is it measurable in experiments or derivable from physical principles? I will consider some actual and hypothetical experiments that help to give insight into the proper role of nonlocality in continuum mechanics and the mechanics of defects. 


\title{
Weak form of bond-associated peridynamics for finite deformation elasticity and rupture in rubber-like materials
}

\author{
Erdogan Madenci \\ The University of Arizona \\ Co-Authors: D. Behera and P. Roy
}

This study considers finite elastic deformation and damage in rubber-like materials under quasi-static loading conditions. The peridynamic equilibrium equation is derived based on the Neo-Hookean hyperelastic model under the assumption of incompressibility. The nonlocal deformation gradient tensor is computed in a bond-associated domain of interaction using the PD differential operator. It is free of oscillations and spurious zero energy modes commonly observed in the PD correspondence models. Also, it permits the direct imposition of natural and essential boundary conditions. The validity of this approach is demonstrated through simulations of experiments concerning progressive damage and rupture in polymers undergoing large elastic deformation.

\section{References}

[1] S. A. Silling, M. Epton, O. Weckner, J. Xu, and E. Askari. Peridynamic states and constitutive modeling. Journal of Elasticity, 88:151-184, 2007.

[2] E. Madenci, M. Dorduncu, A. Barut, and N. Phan. Weak form of peridynamics for nonlocal essential and natural boundary conditions. Computer Methods in Applied Mechanics and Engineering, 337:598-631, 2018.

[3] E. Madenci, M. Dorduncu, N. Phan, and X. Gu. Weak form of bond-associated non-ordinary state-based peridynamics free of zero energy modes with uniform or non-uniform discretization. Engineering Fracture Mechanics, 218:106613, 2019.

[4] E. Madenci, A. Barut, and M. Doruncu. Peridynamic Differential Operator for Numerical Analysis. Springer, NY, 2019. 


\title{
Hybrid FEM and peridynamic simulation of hydraulic fracture propagation in saturated porous media
}

\author{
Ugo Galvanetto
}

The University of Padua

\section{Co-Authors: T. Ni, F. Pesavento, M. Zaccariotto, F. Scabbia, and B. A. Schrefler}

This paper presents a novel hybrid modeling approach for simulating multi-physics problems involving fracture. The new computational method is applied to hydraulic fracture propagation in saturated porous media: Ordinary State based Peridynamics is used to describe the behavior of the solid phase, including crack propagation, while Classical Continuum Mechanics is used to describe the fluid flow and to evaluate the pore pressure. Classical Biot poroelasticity theory is adopted [1,2]. The fluid pressure is applied as an internal force to the solid Peridynamic grid, which deforms and may crack under increasing fluid pressure. Crack propagation changes the porosity of the material and the volume in which the fluid is free to move. This information is continuously transferred between the two fields (solid and fluid) to solve the coupled problem. The accuracy of the proposed approach is initially verified by comparing its results with the exact solutions of two examples. Subsequently we will present the solution of several pressure- and fluid-driven crack propagation examples. The phenomenon of fluid pressure oscillation is observed in the fluid-driven crack propagation examples, which is consistent with previously obtained experimental and numerical data [3]. All the presented examples illustrate the capability of the proposed approach to solve problems of hydraulic fracture propagation in saturated porous media. Finally we observe that the solid is completely discretised with a PD based approach, the fluid with a CCM based method and the mentioned 'coupling' is a physical coupling involving fluid-structure interaction; that is not to be confused with the numerical coupling [4] often cited in the Peridynamic literature.

\section{References}

[1] T. Ni, F. Pesavento, M. Zaccariotto, U. Galvanetto, Q. Zhu, and B. A. Schrefler. Hybrid FEM and Peridynamic simulation of hydraulic fracture propagation in saturated porous media. Submitted for publication, 2019.

[2] R. W. Lewis and B. A. Schrefler. The Finite Element Method in the Static and Dynamic Deformation and Consolidation of Porous Media. John Wiley, 1998.

[3] T. D. Cao, F. Hussain, and B. A. Schrefler. Porous media fracturing dynamics: stepwise crack advancement and fluid pressure oscillations. Journal of the Mechanics and Physics of Solids, 111:113-133, 2018.

[4] T. Ni, M. Zaccariotto, Q.-Z. Zhu, and U. Galvanetto. Coupling of fem and ordinary state-based peridynamics for brittle failure analysis in 3d. Mechanics of Advanced Materials and Structures, 2019. 


\title{
The performance of peridynamic and phase-field models in dynamic brittle fracture
}

\author{
Florin Bobaru \\ University of Nebraska-Lincoln \\ Co-Authors: J. Mehrmashhadi and M. Bahadori
}

We evaluate the performance of three different computational models (an in-house meshfree implementation of peridynamics [1], a discontinuous-Galerkin implementation of peridynamics available in LS-DYNA [2], and a phase-field model implemented in COMSOL, similar to the implementation in [3]) on dynamic brittle fracture in soda-lime glass induced by impact. The evaluation is made against some recent experimental data from [4]. The three models show different crack branching patterns, with the angle of branching being the most significant difference. The fracture pattern predicted by the peridynamic model using the meshfree discretization matches the experimental observations, including some very fine details: small twists in the crack paths as the two crack branches approach the far-right boundary of the sample. The results from the LS-DYNA's Discontinuous Galerkin implementation of peridynamics show spurious/secondary crack branching events, likely due to the way damage growth is implemented in this model. With the phase-field model, the crack branching angle is significantly smaller than in reality and damage continues to "diffuse" into the body after the crack has passed a certain location. A method to prevent this continuous damage expansion in phase-field models of dynamic fracture might be able to improve the phase-field-based results.

\section{References}

[1] F. Bobaru and G. Zhang. Why do cracks branch? A peridynamic investigation of dynamic brittle fracture. International Journal of Fracture, 196:59-98, 2015.

[2] B. Ren, C. T. Wu, and E. Askari. A 3D discontinuous Galerkin finite element method with the bond-based peridynamics model for dynamic brittle failure analysis. International Journal of Impact Engineering, 99:14-25, 2017.

[3] S. W. Zhou, T. Rabczuk, and X. Y. Zhuang. Phase field modeling of quasi-static and dynamic crack propagation: COMSOL implementation and case studies. Advances in Engineering Software, 122:31-49, 2018.

[4] B. M. Sundaram and H. V. Tippur. Dynamic fracture of soda-lime glass: A full-field optical investigation of crack initiation, propagation and branching. Journal of the Mechanics and Physics of Solids, 120:132-153, 2018. 


\title{
Peridynamic modeling of large deformation and ductile fracture
}

\author{
John T. Foster \\ The University of Texas at Austin \\ Co-Author: M. Behzadinasab
}

Prediction of ductile fracture, which is a prevalent failure mode in most engineering structures, is vital to numerous industries. Despite significant advancements in fracture mechanics, ductile fracture modeling has remained a challenging task and a continuing area of research. The peridynamic theory has attracted broad interest in recent years, for its innovative approach for simulating material damage. While peridynamics has been largely utilized to simulate cracking events in brittle materials, its ability in predicting ductile failure remains unclear.

We recently conducted a rigorous investigation into the capabilities of peridynamics in simulating ductile fracture in metallic alloys. The third Sandia Fracture Challenge, as a true blind prediction challenge, was employed in the examination, where the state of the art of peridynamic modeling of ductile fracture was implemented to predict deformations and failure of an additively manufactured metal, with a complex geometry, under the dynamic tensile experiments performed by Sandia National Laboratories. Following the participation in the challenge, while our modeling approach led to qualitatively good results and a correctly predicted crack path, it underpredicted the load-carrying capacity of the structure and simulated an early fracture. Our post-experiment analysis identifies the main sources of discrepancy between the blind simulations and experiments to be (1) material instabilities associated with the finite deformation peridynamic model and (2) unreliability of a Lagrangian peridynamic framework in solving problems involving extremely large deformation and extensive damage.

To address the aforementioned issues, a bond-associated, semi-Lagrangian, constitutive correspondence, peridynamic framework is proposed, in which peridynamic material point interactions depend only on their current properties (e.g. position and stress values) in the deformed configuration, and a rate-based approach is utilized to advance the state of material. A nonlocal version of the velocity gradient is presented to determine the Cauchy stress rate, using local constitutive theories, as an intermediate quantity in computing peridynamic bond forces. A bond-associated, correspondence damage modeling is introduced by using the bond-associated internal properties, e.g. stress and strain values, to incorporate classical failure criteria within the peridynamic framework. The new theory is employed to revisit the Sandia Fracture Challenge problem. Our results indicate that the new approach significantly improves the peridynamic predictions of large deformation and ductile fracture. 


\title{
Convergence studies in meshfree peridynamic wave and crack propagation
}

\author{
Pablo Seleson \\ Oak Ridge National Laboratory \\ Co-Authors: M. Pasetto and Y. John
}

Peridynamics is a nonlocal reformulation of classical continuum mechanics suitable for material failure and damage simulation. Governing equations in peridynamics are based on spatial integration rather than spatial differentiation, allowing natural representation of material discontinuities, such as cracks. A meshfree approach proposed in [1] has been demonstrated to be an effective discretization method for large-scale engineering simulations, particularly those involving large deformation and complex fractures. However, a robust quantitative assessment of the performance of this meshfree method, particularly in fracture scenarios, is lacking. In [2], the convergence of meshfree numerical solutions of static peridynamic problems has been investigated. Related convergence studies for peridynamic wave propagation problems appeared in [3]. In this talk, we will discuss recent convergence studies of wave propagation and extensions to dynamic crack propagation in meshfree peridynamic simulations.

\section{References}

[1] S. A. Silling and E. Askari. A meshfree method based on the peridynamic model of solid mechanics. Computers $\mathcal{E}$ Structures, 83:1526-1535, 2005.

[2] P. Seleson and D. J. Littlewood. Convergence studies in meshfree peridynamic simulations. Computers and Mathematics with Applications, 71:2432-2448, 2016.

[3] P. Seleson and D. J. Littlewood. Numerical tools for improved convergence of meshfree peridynamic discretizations. Handbook of Nonlocal Continuum Mechanics for Materials and Structures, G. Voyiadjis (ed.), Springer, Cham, 2018. 


\title{
Nonlocal brittle fracture: computational analysis and convergence to LEFM
}

\author{
Robert Lipton \\ Louisiana State University \\ Co-Author: P. K. Jha
}

We introduce a state based peridynamic model for calculating dynamic fracture. The force interaction is derived from a double well strain energy density function, resulting in a non-monotonic material model. The material properties change in response to evolving internal forces and fracture emerges from the model. The model can be viewed as a regularized fracture model. In the limit of zero nonlocal interaction the model recovers a sharp crack evolution characterized by the classic Griffith free energy of brittle fracture with elastic deformation satisfying the linear elastic wave equation off the crack set, zero traction on crack faces and the kinetic relation between crack tip velocity and crack driving force given in $[1,4,6,7]$, see $[3,5]$. We complete the talk with a priori convergence rates for the numerical simulation and several computational examples [2]. This research is funded through ARO Grant W911NF1610456.

\section{References}

[1] L. B. Freund. Energy flux into the tip of an extending crack in an elastic solid. Journal of Elasticity, 2:341-349, 1972.

[2] P. K. Jha and R. P. Lipton. Numerical convergence of finite difference approximations for state based peridynamic fracture models. Computer Methods in Applied Mechanics and Engineering, 351:184-225, 2019.

[3] P. K. Jha and R. P. Lipton. In preparation, 2020.

[4] B. V. Kostrov and L. V. Nikitin. Some general problems of mechanics of brittle fracture. Archiwum Mechaniki Stosowanej, 22:749-775, 1970.

[5] R. P. Lipton and P. K. Jha. Classic dynamic fracture recovered as the limit of a non-local peridynamic model: The single edge notch in tension. ArXiv for Mathematics, ArXiv:1908.07589v4 [math.AP] 8 Nov 2019.

[6] L. I. Slepyan. Models and Phenomena in Fracture Mechanics, Foundations of Engineering Mechanics, Springer-Verlag, Berlin, 2002.

[7] J. R. Willis. Equations of motion for propagating cracks. The Mechanics and Physics of Fracture, The Metals Society, 57-67, 1975. 


\title{
A review of benchmark experiments for the validation of peridynamics models
}

\author{
Patrick Diehl \\ Louisiana State University
}

Co-Authors: S. Prudhomme and M. Lévesque

Peridynamics (PD), a non-local generalization of classical continuum mechanics (CCM) allowing for discontinuous displacement fields, provides an attractive framework for the modeling and simulation of fracture mechanics applications. However, PD introduces new model parameters, such as the so-called horizon parameter. The length scale of the horizon is a priori unknown and needs to be identified. Moreover, the treatment of the boundary conditions is also problematic due to the non-local nature of PD models. It has thus become crucial to calibrate the new PD parameters and assess the model adequacy based on experimental observations. The objective of the present paper is to review and catalog available experimental setups that have been used to date for the calibration and validation of peridynamics. We have identified and analyzed a total of 39 publications that compare PD-based simulation results with experimental data. In particular, we have systematically reported, whenever possible, either the relative error or the R-squared coefficient. The best correlations were obtained in the case of experiments involving aluminum and steel materials. Experiments based on imaging techniques were also considered. However, images provide large amounts of information and their comparison with simulations is in that case far from trivial. A total of six publications have been identified and summarized that introduce numerical techniques for extracting additional attributes from peridynamics simulations in order to facilitate the comparison against image-based data.

\section{References}

[1] P. Diehl, S. Prudhomme, and M. Lévesque. A review of benchmark experiments for the validation of peridynamics models. Journal of Peridynamics and Nonlocal Modeling, 1:14-35, 2019. 


\title{
Peri-Net: Analysis of crack patterns using deep neural networks
}

\author{
Guang Lin
}

Purdue University

In this talk, I will introduce convolutional neural networks designed to predict and analyze damage patterns on a disk resulting from molecular dynamic (MD) collision simulations. The simulations under consideration are specifically designed to produce cracks on the disk and, accordingly, numerical methods which require partial derivative information, such as finite element analysis, are not applicable. These simulations can, however, be carried out using peridynamics, a nonlocal extension of classical continuum mechanics based on integral equations which overcome the difficulties in modeling deformation discontinuities. Although this nonlocal extension provides a highly accurate model for the MD simulations, the computational complexity and corresponding run times increase greatly as the simulations grow larger. We propose the use of neural network approximations to complement peridynamic simulations by providing quick estimates which maintain much of the accuracy of the full simulations while reducing simulation times by a factor of 1500 . We propose two distinct convolutional neural networks: one trained to perform the forward problem of predicting the damage pattern on a disk provided the location of a colliding object's impact, and another trained to solve the inverse problem of identifying the collision location, angle, velocity, and size given the resulting damage pattern. 


\title{
Stochastic modeling for uncertainty quantification in fracture mechanics
}

\author{
Johann Guilleminot \\ Duke University
}

This talk is focused on stochastic modeling in fracture mechanics for quasi-brittle, heterogeneous materials. The macroscopic behavior of such materials can be strongly affected by subscale variability, including the effects of both microstructural randomness and spatial variations inelastic and fracture properties. In this talk, we present recent advances related to the modeling of spatial, multiscale-informed variability in elastic and fracture properties, and its impact on the macroscopic response $[1,2]$. We also discuss how fine scale regularity can be leveraged to achieve dimensionality reduction and augment datasets obtained from simulations or physical experiments [3].

\section{References}

[1] D.-A Hun, J. Guilleminot, J. Yvonnet, and M. Bornert. Stochastic multiscale modeling of crack propagation in random heterogeneous media. International Journal for Numerical Methods in Engineering, 119:1325-1344, 2019.

[2] T. Hu, J. Guilleminot, and J. Dolbow. A phase-field model of fracture with traction-free crack surfaces: Application to soil desiccation and thin-film fracture. Submitted, 2019.

[3] J. Guilleminot and J. Dolbow. Data-driven enhancement of fracture paths in random composites. Mechanics Research Communications, available online 16 November, 2019. 


\title{
Experiments in fracture mechanics
}

\author{
Krishnaswamy Ravi-Chandar \\ The University of Texas at Austin
}

One hundred years after Griffith's landmark paper on the theory of rupture, the problem of fracture remains of significant interest. While much success has been achieved in the reliability assessment of structures with cracks, much remains to be done in terms of predictive assessment of failure. In this talk, I will focus on the crucial role played by experiments in all aspects of the development of the theory of rupture, providing physical insight and detailed measurements to challenge and to stimulate modeling and simulation methods. Specifically, I will describe a selection of fracture mechanics experiments, encompassing quasi-static and dynamic fracture as well as nucleation of damage and cracks in brittle and ductile materials. Leveraging on the advances in experimental measurement, I will attempt to pose challenges for proper validation of modeling and simulation methods. 


\title{
Experimental identification of dynamic crack branching precursors in soda-lime silicate glass
}

\author{
Hareesh Tippur \\ Auburn University \\ Co-Author: S. Dondeti
}

The dynamic fracture of high-stiffness and low-toughness materials such as soda-lime silicate glass (SLSG) involves crack initiation and growth prior to branching, underlying mechanics of which is not yet understood. Addressing this issue using full-field optical techniques have faced numerous spatio-temporal challenges since crack speeds in this material reach excess of $1500 \mathrm{~m} / \mathrm{s}$ and are accompanied by highly localized sub-micron scale deformations. Recent work by the authors have shown that transmission-mode Digital Gradient Sensing method [1,2] is capable of overcoming many of these challenges to visualize crack-tip fields in the whole field and quantify fracture parameters associated with each of the phases of crack growth in SLG plates. In this work, time-resolved stress gradient and stress measurements in SLSG plates of two different geometries subjected to dynamic wedge-loading are performed. The LEFM-based precursors extracted from optical measurements leading up to single or sequential/cascading branch formations in SLSG are reported. The identification of precursors are based on crack velocity, stress intensity factors, higher order coefficients of the asymptotic crack tip fields and non-dimensional parameters based on a combination of these. Fracture surface roughness and its features are also separately quantified using high resolution post-mortem examination and corroboration with optically measured quantities.

\section{References}

[1] B. M. Sundaram and H. V. Tippur. Full-field measurement of contact-point and crack-tip deformations in soda-lime glass. Part-II: Stress wave loading. International Journal of Applied Glass Science, 9:123-136, 2018.

[2] B. M. Sundaram and H. V. Tippur. Dynamic fracture of soda-lime glass: A quantitative full-field optical investigation of crack initiation, propagation and branching. Journal of the Mechanics and Physics of Solids, 120:132-153, 2018. 


\title{
On the identification and validation of fracture mechanics models for curved crack in mortar
}

\author{
Francois Hild \\ École Normale Supérieure Paris-Saclay \\ Co-Authors: R. Vargas, A. Tsitova, F. Bernachy-Barbe, B. Bary, and R. B. Canto
}

In this presentation, an approach to defining the path of a curved crack in a single edge notched specimen with gray level residuals extracted from digital image correlation [1] is followed by the calibration of the parameters of a cohesive zone model [2,3]. Only the experimental force is used in the cost function minimized in finite element model updating. The displacement and gray level residual fields allow for the validation of the calibrated parameters. Last, a phase field model is probed with the previously calibrated parameters.

\section{References}

[1] F. Hild, A. Bouterf, and S. Roux. Damage measurements via DIC. International Journal of Fracture, 191:77-105, 2015.

[2] K. Park, G. H. Paulino, and J. R. Roesler. A unified potential-based cohesive model of mixed-mode fracture. Journal of the Mechanics and Physics of Solids, 57:891-908, 2009.

[3] K. Park and G. H. Paulino. Computational implementation of the PPR potential-based cohesive model in ABAQUS: Educational perspective. Engineering Fracture Mechanics, 93:239-262, 2012. 


\title{
Full-field experimental measurements in extreme loading environments for property extraction and simulation validation
}

\author{
John Lambros \\ University of Illinois at Urbana-Champaign
}

Numerical simulations, whether based on finite element, boundary element, peridynamics or other methods, have always been intimately linked with companion experiments. Traditionally experiments have been used to provide direct input to numerical simulations for quantities that could not be directly derived from first principles, e.g., elasto-plastic mechanical properties and the like. However, linking simulations with experiments also allowed for indirect extraction of properties that might not have been directly measurable, e.g., strength properties or thermal dependence of properties. In such cases, inverse problem techniques have been used which provide indirect extraction of the desired quantities by minimizing/optimizing some error or objective function. Finally, even for the case of fully-predictive simulations, companion experiments are critical in validating the simulation framework and its assumptions. Early attempts to couple experiments and simulations in these ways were based on the limited data obtained by point measurements (e.g., strain gauges) and were applied to simple or idealized environments (e.g., under slow loading rates and/or at room temperature). However, as full-field optical measurement techniques gained popularity, experimental data of larger portions of a structure/material allowed for much larger amounts of data to be used in these roles (i.e., material input, property extraction, or simulation validation). More recently, with the advent of the optical metrology technique of two-dimensional (2D) Digital Image Correlation (DIC), and now its three-dimensional (3D) extension Digital Volume Correlation (DVC), we can obtain very large data sets of experimental measurements of displacement and strain on the surface or in the interior of an object. In addition, this is increasingly done under extreme conditions involving high loading rate and/or temperature. In this work we will present a series of experimental measurement techniques and approaches both for the inverse numerical extraction of material properties from experiments and the validation of predictive simulations based primarily on the finite element framework. In the first we will present examples on the extraction of cohesive failure laws from both 2D and 3D experimental data. We have employed 2D DIC on materials with complex microstructures, such as Functionally Graded Materials (FGMs) and particulate reinforced composites, and have used gradient-based inverse schemes to extract cohesive failure relations for these materials. We have also employed stereo-vision DIC for the validation of thermomechanical loading simulation results. The validation methodology uses image decomposition techniques together with an error analysis framework to provide confidence levels of the simulations when compared to companion experiments. Examples will be provided for both dynamic loading situations (e.g., dynamic fracture or vibratory loading) and high temperature loading (e.g., thermomechanical fatigue). 


\title{
Interplay of fracture and plasticity in swelling-driven fracture of phase-transforming battery materials
}

\author{
Ata Mesgarnejad \\ Northeastern University \\ Co-Author: A. Karma
}

Due to their tenfold higher storing capacities, silicon and germanium have emerged as a promising replacement for Carbon-based anodes in Li-ion batteries. However, lithiation of both materials results in large $(300 \%)$ volume expansion that results in an amorphization of their crystalline structure. The significant volume expansion causes the anode particles to change shape and drives inelastic deformation, plastic flow, and fracture within the particle. Experimental observations show that lithiation in both materials is reaction controlled; thus, an invading lithiation front is created that is atomically sharp. Crucially, the initial lithiation of crystalline $\mathrm{Si}(\mathrm{c}-\mathrm{Si})$ is highly anisotropic while it remains isotropic for crystalline $\mathrm{Ge}$ and amorphous $\mathrm{Si}$, which has been suggested as a source of its inferior performance. Here we exploit the power of the phase-field approach to describe both the motion of phase boundaries and stress-driven fracture within a self-consistent set of equations to shed light on the failure modes of Si battery anode material. We simulate the crystalline to amorphous phase transformation of Si and Ge using a modified Allen-Cahn model with anisotropic mobility to replicate the experimental observations. The mechanical response due to lithiation-driven swelling of the anode is modeled using neo-Hookean elasticity coupled with finite J-2 plasticity. Finally, we model fracture using a variational phase-field formulation that is able to describe both nucleation and propagation of cracks without interpenetration of crack surfaces under compression. We use our framework to probe deformation and fracture of Si nanopillars due to lithiation over a wide range of yield strengths. Using 1D axisymmetric simulations, we show how the stresses generated in the nanopillar attain a maximum at a critical yield strength. Then using full 2D plane stress simulations of a nanopillar cross-section, we highlight the emergence of plastic instabilities, which augment the stresses. Finally, we combine our results of 2D simulation with 1D stability analysis of our phase-field model and experimental observations of the critical size of nanopillars for failure to estimate the lithiation-driven yield strength of Si and Ge. The results highlight the complex interplay between fracture and plasticity in the failure of the silicon anodic components. In addition, we present the results of 2D and 3D simulations that investigate the non-trivial effects of anode geometry on mechanical stability. 


\title{
Shear fracture of metals during in-plane torsion experiments
}

\author{
Dirk Mohr \\ ETH Zurich
}

\section{Co-Authors: C. C. Roth and V. Grolleau}

The comparison of different simple shear experiments revealed the great potential of the in-plane torsion configuration for characterizing the fracture response of sheet metal. Only the in-plane torsion configuration seems to be able to provide perfectly proportional simple shear loading histories for isotropic materials. State-of-the-art torsion tests are machined free of slits, but a circular groove is introduced to reduce the thickness of the material and to ensure defined strain localization away from the clamped boundaries. However, this configuration tends to limit the observability of the entire shear zone. Here, an enhanced in-plane torsion test is developed using a grooved specimen with full optical access to the specimen surface for DIC measurements and enlarged strain rate range up to several 100/s. Its main feature is a new clamping and loading technique from the inner boundary of the specimen. Validation experiments are performed on specimens extracted from an aluminum alloy and steel sheets of various ductility. The experimental campaign includes proportional loading, cyclic loading and strain rate jump tests to demonstrate the ability of the newly-proposed technique. Full optical access to the specimen surface is used to reveal the effect of the material anisotropy on the strain field along the sheared area. The findings are compared to results from numerical simulations and with other in-plane shear tests. 


\title{
How moving cracks in brittle solids choose their path
}

\author{
Jay Fineberg
}

The Hebrew University of Jerusalem

Co-Authors: L. Rozen-Levy and J. Kolinski

While we have an excellent fundamental understanding of the dynamics of 'simple' cracks propagating in brittle solids, we do not fully understand how the path of moving cracks is determined. Here we experimentally study cracks that propagate between $10-95 \%$ of their limiting velocity within a brittle material. We deflect these cracks by either allowing them to interact with sparsely implanted defects or driving them to undergo an intrinsic oscillatory instability in defect-free media. Dense, high-speed measurements of the strain fields surrounding the crack tips obtained via imaging reveal that the paths selected by these rapid and strongly perturbed cracks are entirely governed by the direction of maximal strain energy density and not by the often assumed principle of local symmetry. This fundamentally important result may potentially be utilized to either direct or guide running cracks.

\section{References}

[1] E. Bouchbinder, T. Goldman, and J. Fineberg. The dynamics of rapid fracture: instabilities, nonlinearities and length scales. Reports on Progress in Physics, 77:046501, 2014.

[2] T. Goldman, A. Livne, and J. Fineberg. Acquisition of inertia by a moving crack. Physical Review Letters, 104:114301, 2010.

[3] A. Livne, E. Bouchbinder, I. Svetlizky, and J. Fineberg. The near-tip fields of fast cracks. Science, 327:1359-1363, 2010. 


\title{
A gradient-damaged theory for fracture of quasi-brittle materials
}

\author{
Lallit Anand
}

Massachusetts Institute of Technology

I will present a gradient-damage theory for fracture of "quasi-brittle" materials under tensile dominated stress states. The theory is developed using the method of virtual-power. The macro- and microforce balances, obtained from the virtual power approach, together with a standard free-energy imbalance equation under isothermal conditions, when supplemented with a set of thermodynamically-consistent constitutive equations provide the governing equations for the theory. The general theory has been specialized to formulate a model for fracture of concrete-a quasi-brittle material of vast importance. We have numerically implemented our theory in a finite element program, and we present results from representative numerical calculations which show the ability of our simulation capability to reproduce the macroscopic load-deflection characteristics as well as crack-paths during failure of concrete in several technically relevant geometries reported in the literature.

\section{References}

[1] S. Narayan and L. Anand. A gradient-damage theory for fracture of quasi-brittle materials. Journal of the Mechanics and Physics of Solids, 129:119-146, 2019. 


\title{
Revisiting nucleation in the phase-field approach to brittle fracture
}

\author{
Oscar Lopez-Pamies \\ University of Illinois at Urbana-Champaign \\ Co-Authors: A. Kumar, B. Bourdin, and G. A. Francfort
}

Twenty years in since their introduction [1], it is now plain that the regularized formulations dubbed as phase-field of the variational theory of brittle fracture of Francfort and Marigo [2] provide a powerful macroscopic theory to describe and predict the propagation of cracks in linear elastic brittle materials under arbitrary quasistatic loading conditions. Over the past ten years, the ability of the phase-field approach to also possibly describe and predict crack nucleation has been under intense investigation. The first of two objectives of this talk is to establish that the existing phase-field approach to fracture at large-irrespectively of its particular version-is fundamentally incomplete to model crack nucleation. This is because the approach is purely energetic and cannot account for one essential ingredient that is not energetic but rather stress-based: the strength of the material. The second objective is to introduce an amendment that renders a phase-field theory capable of modeling crack nucleation in general, be it from large pre-existing cracks, small pre-existing cracks, smooth and non-smooth boundary points, or within the bulk of structures subjected to arbitrary quasistatic loadings. Following Kumar, Francfort, and Lopez-Pamies [3], the central idea is to implicitly account for the presence of the inherent microscopic defects in the material - whose defining macroscopic manifestation is precisely the strength of the material-through the addition of an external driving force in the equation governing the evolution of the phase field. To illustrate the descriptive and predictive capabilities of the proposed theory, the last part of the talk will be devoted to present sample simulations of experiments spanning the full range of fracture nucleation settings.

\section{References}

[1] B. Bourdin, G. A. Francfort, and J. J. Marigo. Numerical experiments in revisited brittle fracture. Journal of the Mechanics and Physics of Solids, 48:797-826, 2000.

[2] G. A. Francfort and J. J. Marigo. Revisiting brittle fracture as an energy minimization problem. Journal of the Mechanics and Physics of Solids, 46:1319-1342, 1998.

[3] A. Kumar, G. A. Francfort, and O. Lopez-Pamies. Fracture and healing of elastomers: A phase-transition theory and numerical implementation. Journal of the Mechanics and Physics of Solids, 112:523-551, 2018. 


\title{
Thermal-conductivity degradation across cracks in coupled thermo-mechanical systems modeled by the phase-field fracture method
}

\author{
Haim Waisman \\ Columbia University
}

Co-Authors: L. Svolos and C. A. Bronkhorst

Dynamic loading of polycrystalline metallic materials can lead to brittle or ductile fracture depending on the loading rates, geometry and material type. Cracks develop rapidly with minimal plasticity and minimal heat dissipation when brittle fracture is observed (e.g. Kalthoff problem under low strain rates). At high strain rates for metallic materials which can accommodate plastic deformation, material instabilities known as shear bands, can occur. Shear bands are narrow localization bands which reduce the stress bearing capacity of the material and act as a precursor to ductile fracture (e.g. cracks that develop rapidly on top of a shear band). A unified model, which has been developed in [1, 2], accounts for the two aforementioned failure processes simultaneously. In this model, the phase-field method is used to model crack initiation and propagation, and is coupled to a temperature dependent visco-plastic model that captures shear bands. In this work, an improvement to the unified model is presented in order to capture more accurately the heat transfer across the fracture surfaces. Specifically, an isotropic degradation of the thermal conductivity is proposed, which couples the thermal diffusion process with the extent of damage across a crack. The closed form solution is derived analytically based on a micro-mechanics void extension model of Laplace's equation. We investigate the behavior of the aforementioned technique on two benchmark problems and show the necessity of such physics-based degradation function in dynamic fracture problems.

\section{References}

[1] C. McAuliffe and H. Waisman. A unified model for metal failure capturing shear banding and fracture. International Journal of Plasticity, 65:131-151, 2015.

[2] C. McAuliffe and H. Waisman. A coupled phase field shear band model for ductile-brittle transition in notched plate impacts. Computer Methods in Applied Mechanics and Engineering, 305:173-195, 2016. 


\title{
Phase-field fracture models for linearized and finite strains
}

\author{
Kerstin Weinberg \\ University of Siegen
}

While cracks are actually sharp two-dimensional hypersurfaces the phase-field fracture approach regularizes the sharp material discontinuities with smooth transitions between broken and unbroken regions. The evolution of the phase-field follows an evolution equation where the driving forces of crack growth are derived from an energy minimization principle, typically based on an Ambrosio-Tortorelli type functional. Modifications allow accounting for the no-healing irreversibility constraint of crack evolution and, especially important, for the asymmetry of fracture, i.e., the fact that cracks only grow under tensile loadings but not under compression. Further modifications consider the evolution problem at finite strains using energy densities, which are polyconvex functions of the deformation $[1,2]$. In this contribution different decompositions of the elastic energy and the pros and cons of variational and ad hoc formulations for the crack driving forces will be discussed. The latter may base on positive principal stresses or strains for example. We compare different models in linearized and in finite elasticity and present recent results on the mathematical analysis for a phase-field model at finite strains, where we formulate the phase-field with energy densities in terms of the modified invariants of the right Cauchy-Green strain tensor. To illustrate the capability of a phase-field fracture approach we present finite element simulations of brittle fracture and compare it to our experimental results. The main challenge of such fracture simulations is that it requires the ability of a numerical method to predict crack nucleation and fracture without stress concentration at a notch or at an initial crack.

\section{References}

[1] C. Bilgen and K. Weinberg. On the crack-driving force of phase-field models in linearized and finite elasticity. Computer Methods in Applied Mechanics and Engineering, 353:348-372, 2019.

[2] C. Bilgen, A. Kopaničáková, R. Krause, and K. Weinberg. A phase-field approach to conchoidal fracture. Meccanica, 53:1203-1219, 2018. 


\title{
Isogeometric and meshfree analysis of hyperbolic phase field fracture
}

\author{
David Kamensky \\ University of California San Diego \\ Co-Authors: G. Moutsanidis and Y. Bazilevs
}

\begin{abstract}
We introduce a phase field fracture formulation that results in a hyperbolic partial differential equation (PDE) for the order parameter distinguishing broken and unbroken phases of the material [1,2]. This PDE can be formally derived from microforce balance theory by associating a microscopic kinetic energy with the order parameter's rate of change. An important practical advantage is that hyperbolic PDEs are more amenable to explicit time stepping, which is almost universally favored in simulations of extreme events like explosions, where small time steps are needed to resolve rapid phenomena. Finite element computations of benchmark problems demonstrate that the properties of the new model are similar to elliptic phase field models (relative to typical modeling errors of current methods), although there is a noticeable rate-toughening effect, which becomes more pronounced as the wave speed is reduced. The model is then implemented into a hybrid meshfree-isogeometric hydrocode to simulate more complicated scenarios and perform qualitative comparisons with experimental results from the literature on blast-induced fracture of PMMA. We conclude with some discussion of ongoing and future work on efficient implementation of isogeometric and meshfree technologies, such as using automatic code generation [3] to rapidly test new formulations.
\end{abstract}

\section{References}

[1] D. Kamensky, G. Moutsanidis, and Y. Bazilevs. Hyperbolic phase field modeling of brittle fracture: Part I-Theory and simulations. Journal of the Mechanics and Physics of Solids, 121:81-98, 2018. [2] G. Moutsanidis, D. Kamensky, J. S. Chen, and Y. Bazilevs. Hyperbolic phase field modeling of brittle fracture: Part II-immersed IGA-RKPM coupling for air-blast-structure interaction. Journal of the Mechanics and Physics of Solids, 121:114-132, 2018.

[3] D. Kamensky and Y. Bazilevs. tIGAr: Automating isogeometric analysis with FEniCS. Computer Methods in Applied Mechanics and Engineering, 344:477-489, 2019. 


\title{
Some recent progress on phase-field modeling: fatigue, r-curves, and large structures
}

\author{
Chad Landis
}

The University of Texas at Austin

\author{
Co-Authors: Y.-S. Lo, A. Anvari, K. Ravi-Chandar, T. J. R. Hughes, and M. J. Borden
}

Over the last few decades, the phase-field approach to fracture has been shown to be a useful tool for modeling complex crack path evolution. Features including the nucleation, turning, branching, and merging of cracks as a result of quasi-static mechanical and dynamic loadings are captured without the need for extra constitutive rules for these phenomena. This presentation will touch on our recent work on the phase-field modeling approach for fatigue crack growth, R-curve behavior for brittle fracture in the presence of plastic flow, and modifications for large-scale structures will be discussed. For fatigue, a modified J-integral will be developed to demonstrate how the phase-field approach can be used to generate Paris-Law type crack growth rates. A steady-state finite element method is then applied to generate fits of the phase-field theory to measured crack growth rate data. Full transient simulations are performed and compared to experimental measurements on samples where crack turning is induced by the presence of a hole in the vicinity of the crack. To model R-curve behavior plasticity is introduced into the formulation and adaptive refinement is used to capture different length scales. Finally, modifications to the damage functions are introduced to allow for the analysis of large scale structures and some issues are identified and discussed. 


\title{
Attempts to validate phase-field fracture for nearly incompressible solids with experimental data
}

\author{
Thomas Wick \\ Leibniz University Hannover \\ Co-Authors: K. Mang, N. Kröger, M. Walloth, and W. Wollner
}

In this presentation, we propose the first steps of validating a phase-field model for nearly incompressible solids. To avoid locking effects therein, we propose a mixed form for the solid displacement equation as formulated in [1]. For the numerical solution, we adopt a fully-coupled approach, which is implemented in DOpElib [2] and currently extended with adaptive mesh refinement and parallelization using the deal.II-github-phase-field framework [3]. To validate this new phase-field model for rubber-like materials, we compare - in collaboration with the Deutsches Institut für Kautschuktechnologie e. V., Hannover, Germany-experimental data of crack propagation in Carbon Black filled EPDM rubber against our numerical simulations and figure out differences, difficulties and challenges.

\section{References}

[1] K. Mang, T. Wick, and W. Wollner. A phase-field model for fractures in nearly incompressible solids. Computational Mechanics, published online, 2019.

[2] DOpElib: The Differential Equation and Optimization Environment, www.dopelib.net.

[3] T. Heister and T. Wick. Parallel solution, adaptivity, computational convergence, and open-source code of 2D and 3D pressurized phase-field fracture problems. Proceedings in Applied Mathematics and Mechanics, 18:e201800353, 2018. 
A-28 
POSTER PRESENTATIONS

Simulating fractures in rubber - one step further with phase-field modeling

\author{
Katrin Mang \\ Leibniz University Hannover
}

Co-Authors: Andreas Fehse, Nils-Hendrik Kröger, and Thomas Wick

\title{
Full field in-situ Digital Image Correlation measurements of Mode I and Mode II crack
} growth for polymeric materials

\author{
Ilyass Tabiai \\ Polytechnique Montréal
}

Co-Authors: Martin Lévesque, Daniel Therriault, Damien Texier, and Philippe Bocher

Asymptotically compatible reproducing kernel collocation and meshfree integration for Peridynamic Navier equation

Yu Leng

The University of Texas at Austin

Co-Authors: Xiaochuan Tian, Nathaniel Trask, and John T. Foster

Fractional derivatives and viscoelasticity

\author{
Mary Vaughan \\ Iowa State University
}

Co-Authors: Pablo Raúl Stinga and Pablo Seleson

Two-dimensional reduction of 3D axisymmetric peridynamic models

Debdeep Bhattacharya

George Washington University

Co-Authors: Pablo Seleson and Jeremy Trageser 


\title{
Peridynamic modeling of large deformation and ductile fracture
}

Masoud Behzadinasab

Brown University

Co-Authors: John Foster and Yuri Bazilevs

\section{Convergence in peridynamic fracture modeling}

\author{
Marco Pasetto \\ Oak Ridge National Laboratory \\ Co-Author: Pablo Seleson
}

Fast algorithm for peridynamics and nonlocal models in heterogeneous media

Xiaochuan Tian

The University of Texas at Austin

Co-Author: Bjorn Engquist

Dynamic fracture modeling with peridynamics

Sahir Butt

Ruhr University Bochum

Co-Author: Günther Meschke 
APPENDIX B. PARTICIPANTS' FEEDBACK 



\section{PERIDYNAMICS}

1. What are the biggest challenges and opportunities in peridynamics fracture modeling?

044401b3

- Biggest challenge is in calibration of the material properties even for elastic, brittle systems.

- Nucleation of cracks.

- Ductile fracture.

7e60a83f

- Fracture criteria incorporating mechanism information.

- Demonstrating convergence with respect to discretization.

- Coupling to classical continuum in dynamics setting.

e63e52ee

- What experimental observations should a good model (phase field or peridynamic) provide:

(a) For amorphous brittle materials, we should be able to predict the oscillatory instability at very high velocities-(how) is nonlinear elasticity linked to this instability? Can spontaneous microbranching from $0.3 c_{R}$ ( 0.3 of the Rayleigh wave speed) or so and higher be observed?

(b) Question: The cohesive zone-What is its form and what does the form depend on? Can nontrivial cohesive zone behavior or dynamic behavior (e.g. flash heating) take place? Under what condition?

(c) 3D fracture. Can the experimentally observed facetting and nontrivial topology be observed? How does a crack front behave under large (finite) perturbations? Can the crack front break up into separate, stable, fracture planes?

(d) What is the effect of the interaction of the crack front with either rigid or soft particles? Can the resulting behavior and crack propagation direction be predicted?

$2 \mathrm{f} 8347 \mathrm{e} 0$

- In my opinion, the biggest challenge while modeling fracture using peridynamics is to understand all the influences of the nonlocal horizon on the fracture process and to interpret it in terms of a physical length scale associated with the fracture process.

24320515

- What is the horizon? A discretization parameter or a material property? How is the horizon determined?

- Ductile fracture models.

- Application of local boundary conditions from the experiment in a non-local fashion.

$2 \mathrm{f} 8347 \mathrm{e} 0$

- Extracting experimentally-measurable quantities of interest (e.g., crack length or speed) in robust ways.

- Defining and applying Neumann boundary conditions.

- Contact mechanics.

${ }^{*}$ Participants were assigned a code to maintain their anonymity. 
$1 \mathrm{e} 041544$

- Challenges in peridynamics fracture modeling:

(a) Computational cost: the so-called meshfree discretization (based one one-point Gaussian spatial integration) used for modeling fracture in peridynamics is costly. Discretization methods able to reduce the cost would allow to scale simulations to larger samples while maintaining the resolution necessary for reasonable predictions of evolution of failure and damage.

- Opportunities in peridynamics fracture modeling:

(a) Mixed-mode fracture: while in most predominately Mode-I driven fracture peridynamics does an excellent job predicting the behavior, Mode-II dominated fracture has not received enough attention. This is an important topic, especially since it directly relates to a number of topics listed next.

(b) Fracture and damage at an interface: most fracture criteria used in peridynamic models are for Mode-I dominated cases. At an interface, especially in dynamic problems, Mode-II loading becomes relevant, and sometimes predominant. Validation of peridynamic models for these cases is still needed.

(c) Adhesive versus cohesive fracture: peridynamic models are needed to determine the evolution of cracks in bonded materials, adhesive joints, etc.

(d) Partially-homogenized models for materials with microstructure: material microstructure plays a critical role in crack initiation and subsequent fracture/damage evolution (e.g., fiber-reinforced composites, metal-ceramic composites, and additively manufacture materials). Multiscale models that fully resolve the small scale are usually expensive. Partial homogenization (maintaining some information about the microstructure) would be significantly cheaper to use and still provide realistic evolution of fracture/damage at the larger scale.

(e) Multi-physics fracture: stress corrosion cracking, diffusion-induced fracture, thermally-induced cracks, ductile-brittle transition.

2. What do we need to experimentally validate peridynamic fracture models?

$044401 b 3$

- A collection of carefully documented challenge problems spanning a range of ingredients such as mixed-mode, quasi-brittle (cohesive zone), dynamics, and ductile fracture (Sandia challenges).

fe6a43f8

- From my recent studies on brittle fracture, I have noticed that there is a region (X) of concentrated kinetic energy around a propagating crack tip which increases its size with respect to the size of the peridynamic horizon. So I think, if there is a possibility to experimentally monitor the spatiotemporal evolution of the size of this region $(\mathrm{X})$ where kinetic energy is getting concentrated around a propagating crack tip (the size of this region $(\mathrm{X})$ will be a function of crack speed). A minimum size of this region (Xmin, which would be a material parameter) can be defined with respect to a "minimum observed crack speed" and this size (Xmin) would provide (with some scaling) a lower bound for the horizon size.

$2 \mathrm{f} 8347 \mathrm{e} 0$

- For validating bond-based peridynamics in particular, there is a dearth of experimental results using materials with Poisson's ratio sufficiently close to 0.25 . 
$1 \mathrm{e} 041544$

- We have had success validating peridynamic dynamic/quasi-static fracture models in glass, PMMA, and some ceramics against some recent experimental data. Experiments with fully described loading and boundary conditions for brittle and quasi-brittle fracture are needed for further validation of peridynamic models. Samples with different geometries (e.g. interfaces placed at different locations) can lead to significantly different brittle fracture behavior, and testing whether peridynamic models can track that would be a great validation test.

\section{PHASE FIELD MODELING ${ }^{\dagger}$}

1. What are the biggest challenges and opportunities in phase field fracture modeling?

$040601 \mathrm{a} 0$

- Find an experimental way to correlate length scales to microstructures and provide measures for length scales.

- Find critical fracture energy (or fracture toughness) for inhomogeneous materials.

- Fracture experiments in bi- and tri-axial loading states.

- More experiments on ductile fracture with different geometries and boundary conditions.

- Measure temperature and heat transfer in high strain rate ductile fracture.

- Fracture experiments on polymers undergoing large deformations.

- High/low cycle fatigue experiments in polymers and metals (and other materials such as ice).

- Fracture experiments of ice grown in a lab, which are currently important due to climate change.

- Rupture experiments of soft/hard biological tissues.

- Design experiments to model fracture of geomaterials (e.g. validate hydraulic fracture models).

$044401 \mathrm{~b} 3$

- Connection of the phase field length scale to physical processes.

- Nucleation of cracks.

- Ductile fracture.

$041 \mathrm{a} 3722$

- Thorough numerical analysis. We have mathematical results (calculus of variations) and a lot of engineering results. Rigorous numerical analysis is still missing.

\footnotetext{
'Participants were assigned a code to maintain their anonymity.
} 
$224 \mathrm{~d} 87 \mathrm{~d} 4$

- There remain significant challenges in phase field fracture modeling that form an obstacle to widespread adoption.

- Relatively little work has been done on ductile fracture with phase field, something of great interest to the national laboratories. Great work has been done in micromechanically-based damage models that characterize void growth, nucleation, and anisotropy, but the intelligent connection of these models to phase field techniques is unclear. Ductile phase field models that do not include these capabilities are missing important physics.

- Some modeling choices are relatively minor in the theoretical formulation, but remain of utmost importance in determining the fracture physics, such as the tension/compression splits. I have seen little convincing evidence of one split that's valid for all loading cases. It is necessary to have a unified and coherent method for determining where cracks can grow that works in Mode-I, mixed-mode, sheardominated, etc. cases that requires no analyst intervention.

- Despite the simple theoretical formulation, some modeling aspects may require complex solutions. A long-standing aspect of phase field fracture modeling has been to use a maximum historical driving energy to prevent crack reversion. This is energetically inconsistent and may pollute simulation results, but the solutions proposed are things like inequality-constrained solvers and Lagrange multipliers. Unfortunately, this significantly increases the implementation effort, which might put the method out of reach.

- Computational expense remains an issue, due to the need of sufficient resolution of an often-miniscule regularization length scale. Approaches that decouple the length scale parameter from physics might provide a path forward here, but there will likely be limits there too. Integration of multi-scale methods may be a key component for widespread adoption of the phase field method. This too significantly increases the implementation effort.

026f0137

- The biggest opportunity in my opinion is the use of the phase field method to shine light on how the macroscopic fracture toughness of composites emerges from their microstructure.

$7 \mathrm{e} 60 \mathrm{a} 83 \mathrm{f}$

- Mixed model fracture and anisotropic materials/criteria.

$2 \mathrm{cb} 6277 \mathrm{c}$

- Most of the research efforts to date have focused on propagation of fracture, yet without nucleation there is no propagation. Thus nucleation is one of the biggest problems that remain open at present. 
$2 \mathrm{f} 8347 \mathrm{e} 0$

- In fragmentation problems, how does one handle the order-length-scale region of very soft material, which will undergo extreme deformations? Simple element deletion will not satisfy mass conservation. Introducing a small residual stiffness can cause problems with sudden, rapid crack widening in certain dynamic fracture cases.

- How can one robustly extract the crack surface from the phase field solution?

- In some dynamic fracture problems, I have observed widening of the damaged zone over time, eventually resulting in all the material becoming damaged, rather than breaking apart into discrete undamaged fragments.

- The brute force approach of meshing everything with elements smaller than the fracture length scale is untenable for many practical problems, so robust strategies for on-the-fly local refinement are needed.

- Although various solutions have been proposed in the literature, there doesn't seem to be a consensus on how best to incorporate phase field models (which typically involve an elliptic PDE) into fullyexplicit hydrocodes used for extreme event simulation.

- Can phase field fracture be discretized in an asymptotically-compatible way? In other words, can it be formulated to converge to classical linear elastic fracture mechanics with the nominal energy release rate while keeping the element size proportional to the length scale?

Of 210354

- In general the cracks are quite smooth in phase field fracture modeling-which is not necessarily physical. Also, the crack path is indeed unknown in most examples-which makes it difficult to call it wrong or right. Another challenge of phase field fracture modeling is the dependence of the computations on many parameters, numerical and modeling ones. There is the regularizing length $l_{0}$ but also mobility/viscosity of evolution, weight of crack driving force, limits for non-reversible phase field evolution*, degradation function, etc.

${ }^{*}$ Most codes use a lower bound to define if there is a crack and to avoid artifacts. The choice of this value makes a difference, in particular in dynamic fracture when a wave cracks the structure.

$1 \mathrm{e} 041544$

- Dynamic brittle fracture: we (and others) have observed a non-physical continuous "bleeding"/progression of damage into the material after cracks pass by.

2. What do we need to experimentally validate phase field fracture models?

$044401 \mathrm{~b} 3$

- Should be the same as for peridynamics!

$041 \mathrm{a} 3722$

- No idea. Experimental people have to tell the simulations people (like me) what is currently possible to measure. On the other hand, the simulation people must tell which simulations $(2 \mathrm{D}, 3 \mathrm{D}, \ldots)$ are reliable so far. Many results in the literature are there, but most of them are not reliable.

026f0137

- Phase field models for brittle and ductile fracture are both experimentally validated for many materials. Further validation is needed for more complex materials, for example brittle gels under finite deformation. 
$2 \mathrm{cb} 6277 \mathrm{c}$

- The experimental challenges to obtain measurements of fracture nucleation within the bulk of materials are enormous, yet they are absolutely necessary if we are to construct a truly predictive theory.

2f8347e0

- For both phase field and peridynamic models, there are already many existing experimental results that, at least for the basic brittle case, would be largely sufficient for validation. However, these results are typically not documented in ways that make them useful for validation. It would be useful if experimental teams developed (or found collaborators with) enough fluency in mathematical models and computational methods that the needs of these models and methods could be used as a "checklist" for publishing experimental results.

- How do we distinguish clearly between physical instabilities which would occur in a perfectlyhomogeneous material and "random" behavior that is really the result of random spatial fluctuations in material properties? And, for the latter, how do we characterize such fluctuations in experimental samples, such that statistically-similar fluctuations can be included in ensembles of computations?

- It would be valuable to have quality high-speed footage of dynamic fracture under controlled experimental conditions.

Of210354

- Experiments are essential for validation of computational results. In particular we need experiments with simple geometry and with clear boundary conditions and loadings. It would be helpful to quantify other dissipative effects which come along with fracture like vibrations of the fragments and a possible temperature rise.

\section{EXPERIMENTAL FRACTURE MECHANICS}

1. What are the biggest challenges and opportunities in experimental fracture research?

$040601 \mathrm{a} 0$

- Trustworthy and reproducible experiments for a range of problems and materials that I mentioned above.

$044401 b 3$

- There is not much funding in this area anymore! Perhaps developing a MURI type effort combining experiments with predictive methods could be a driver.

- Dynamic experiments are difficult, expensive; but improved diagnostic methods have been developed and this presents a new opportunity.

6172ab11

- Current lack of measurement tools to study fracture mechanics of high-stiffness and low-toughness materials such as ceramics, cementitious and geomaterials is both a challenge and an opportunity.

${ }^{\ddagger}$ Participants were assigned a code to maintain their anonymity. 
24320515

- I think that accessing raw experimental data is a challenge right now. An opportunity there could be Open Data to make the raw experimental data accessible. This would make it easier to access a set of the same experiment to not only fit the peridynamic/phase field model to one experiment and validate the fitted model against one experiment.

Of 210354

- For crack growth it is very difficult to design experiments with reproducible conditions. Crack growth is always a stability problem and a slight perturbation results in a strong variation of results. Additionally, crack growth is usually fast. Here it is very difficult to measure forces and deformation, and again a slight variation in initial conditions may result in different results and crack patterns.

2. How fracture modeling could help experimental fracture research?

$040601 \mathrm{a} 0$

- Allow us to validate our numerical fracture models, which would then be useful for prediction and design of new materials and structures.

$044401 \mathrm{~b} 3$

- Experiments and modeling should go hand-in-hand in order to make progress; early work on dynamic fracture demonstrates this well.

- Can simulations provide additional insight leveraging on experimental measurements? Right now, it appears that the simulations are looking towards experiments primarily for validation of the methods.

$6172 \mathrm{ab} 11$

- One cannot perform unlimited number of experiments at different scales using the same approach to obtain intelligible results. Fracture modeling could greatly mitigate this issue.

026f0137

- Experiments should be guided by simulations. Simulations and experiments should be combined in cycles:

1. Experimental observation should be tried to be explained by simulations.

2. Fundamental theoretical hypothesis should be drawn from simulations.

3. Based on those hypothesis new experimental specimen/procedures should be designed with falsifiable outcomes.

4. Go to 1 .

Because of the financial and time constraints usually this process is broken at 2.

Of 210354

- Numerical calculations are much more reproducible than experimental results. This could be used to design experiments. Here in particular simple specimens and loading conditions are of advantage because they allow extensive parametric studies. This, however, requires a predictive numerical simulation which is not yet available in general, e.g. for finite deformations and dynamic fracture. 


\section{MACHINE LEARNING AND UNCERTAINTY QUANTIFICATION $\$$}

1. What are the biggest challenges and opportunities in machine learning $(M L)$ for computational and experimental fracture mechanics?

$044401 b 3$

- Not sure. We don't seem to have large data sets.

6172ab11

- For experimental fracture mechanics, particularly dynamic fracture, producing a large number of measurements needed to train the ML algorithm is a challenge.

026f0137

- Stochastic nature of emergence of macro fracture behavior in composites is a perfect opportunity for ML. Phase field and peridynamics simulation of sufficient size nowadays can be done to create the basis big data needed for ML.

24320515

- From my perspective for experiments, we do not have enough experimental data for the ML process. For simulations, since peridynamics and phase field are computational expensive methods, the challenge would be to produce a large set of complicated geometries, e.g. three-dimensional simulations.

2. What are the biggest challenges and opportunities in uncertainty quantification $(U Q)$ for computational and experimental fracture mechanics?

$040601 \mathrm{a} 0$

- How to validate and come up with bounds for UQ of fracture systems. What will be considered a "reasonable" bound?

- Develop efficient stochastic solvers and reduced models that can handle real multiphysics systems.

- Develop UQ for multiphysics, multiscale and multidomain systems with fracture.

- How to present UQ analyses of a system with many-parameter uncertainties in a meaningful/useful way.

- Fracture abruptly changes the system. Develop appropriate statistics for those extreme cases.

e69c1355

- Space-time representations of relevant fields, at relevant scales.

- Statistical identification and validation methodologies.

- Fast stochastic solvers.

- Common language and accessible tools.

$044401 \mathrm{~b} 3$

- UQ can only progress when models are quantitative and predictive; it appears that most numerical methods are able to reproduce qualitatively. I think the biggest challenge is in establishing the predictive capability of the numeric.

\footnotetext{
${ }^{\S}$ Participants were assigned a code to maintain their anonymity.
} 
$6172 \mathrm{ab} 11$

- Tracking and integrating all the uncertainties across multiple aspects of a fracture experiment starting from material and geometric variability to measured load or displacement variability to methodologies (e.g., interferometry or DIC) to data extraction (say, velocity and SIF) is prohibitively challenging.

026f0137

- More often than not it is not easy to isolate the main mechanism responsible for observed failure behavior to distinguish a good observable for the system.

24320515

- Since we have some uncertainty in the material, e.g. defects or influence of drying in concrete, or in the design of the experiment, doing the same experiments a few times will not result in the exact same result. From my opinion the challenge here is to address all these parameters in the UQ model.

\section{GENERAL CONSIDERATIONS}

1. What would be good experiments to compare phase field and peridynamic fracture models? Is such an experimental data available?

026f0137

- There are a good number of basic ones (Noorumohamaed), some of the Bittencort experiments, Hertzian cone fracture as well as many of Fineberg dynamic experiments.

2. Are current fracture models predictive enough?

$040601 \mathrm{a} 0$

- Current fracture methods are predictive in some simple cases (e.g. linear elastic with simple geometry/boundary conditions) but are yet to be predictive in the range of problems I mentioned above.

$044401 \mathrm{~b} 3$

- There is an engineering theory and fracture based designs are routinely used. So, LEFM is predictive. Can't say the same for nonlinear fracture problems.

$041 \mathrm{a} 3722$

- Definitely no. There are too many unknowns, material and model parameters. Often, mesh convergence studies do not yield convergence in quantities of interest. Constitutive models are often unclear and hold for some configurations while not for others.

026f0137

- Yes, phase field has proven very robust in predicting crack paths. There are opportunities in nonlinear and inelastic materials as well as multiphysics fracture problems.

\footnotetext{
IParticipants were assigned a code to maintain their anonymity.
} 
$1 \mathrm{e} 041544$

- I think that peridynamics has made some great strides in predicting fracture: dynamics, quasi-static, brittle, quasi-brittle, and ductile. Unfortunately, no commercial code has implemented and sufficiently tested peridynamic models for reliable fracture prediction. Our tests with LS-DYNA's discontinuous Galerkin implementation shows that this discretization might not be the proper one for modeling dynamic brittle fracture (crack branching, etc.). 